\title{
Small scale density variations of electrons and charged particles in the vicinity of polar mesosphere summer echoes
}

\author{
M. Rapp ${ }^{1}$, F.-J. Lübken ${ }^{1}$, and T. A. Blix ${ }^{2}$ \\ ${ }^{1}$ Leibniz Institute of Atmospheric Physics, Schlossstr. 6, 18225 Kühlungsborn, Germany \\ ${ }^{2}$ Norwegian Defence Research Establishment, Kjeller, Norway
}

Received: 22 May 2003 - Published in Atmos. Chem. Phys. Discuss.: 4 July 2003

Revised: 1 September 2003 - Accepted: 5 September 2003 - Published: 16 September 2003

\begin{abstract}
We present small scale variations of electron number densities and particle charge number densities measured in situ in the presence of polar mesosphere summer echoes. It turns out that the small scale fluctuations of electrons and negatively charged particles show a strong anticorrelation down to the smallest scales observed. Comparing these small scale structures with the simultaneously measured radar signal to noise profile, we find that the radar profile is well described by the power spectral density of both electrons and charged particles at the radar half wavelength (=the Bragg scale). Finally, we consider the shape of the power spectra of the observed plasma fluctuations and find that both charged particles and electrons show spectra that can be explained in terms of either neutral air turbulence acting on the distribution of a low diffusivity tracer or the fossil remnants of a formerly active turbulent region. All these results are consistent with the theoretical ideas by Rapp and Lübken (2003) suggesting that PMSE can be explained by a combination of active and fossil neutral air turbulence acting on the large and heavy charged aerosol particles which are subsequently mirrored in the electron number density distribution that becomes visible to a VHF radar when small scale fluctuations are present.
\end{abstract}

\section{Introduction}

Polar mesosphere summer echoes (PMSE) are very strong radar echoes from around the summer polar mesopause and were first observed in the VHF wavelength range (typical wavelength $\lambda=6 \mathrm{~m}$ ) about two decades ago (Ecklund and Balsley, 1981). At mesopause heights, radar waves are scattered due to irregularities in the electron number density at the Bragg scale $(=\lambda / 2$ for monostatic radars). The under-

Correspondence to: M. Rapp

(rapp@iap-kborn.de) standing of the physical processes leading to the formation of such small scale structures in the electron gas has turned out to be a longstanding open question. First ideas that the electron number density structures are directly related to neutral air turbulence were soon discarded since it turned out that under typical conditions in the mesopause region a length scale of $3 \mathrm{~m}$ is far in the viscous subrange, where any structures are efficiently destroyed by molecular diffusion (e.g. Lübken et al., 2002). The first breaktrough in the understanding of PMSE was achieved by Kelley et al. (1987) who proposed that the electrons at the polar summer mesopause are low diffusivity tracers due to the presence of large and heavy positive ions such as water cluster ions. If the tracer diffusivity is significantly smaller than the viscosity of air, the fluctuations in the tracer field can extend to much smaller scales than in the neutral gas (Batchelor, 1959). This picture was then refined by Cho et al. (1992) who proposed that the diffusivity reduction should rather be a consequence of the presence of charged ice particles which were known to exist at PMSE altitudes from lidar observations of noctilucent clouds (e.g. von Zahn and Bremer, 1999). The main result of Cho et al.'s theory was that the electron diffusivity should reduce to the diffusivity of the aerosol particles (which can be orders of magnitude less than positive ion diffusivity due to the large aerosol particle mass) provided that the mesospheric charge balance is dominated by the charge concentration of the heavy particles. Significant support to this idea came from observations of deep electron depletions at PMSE altitues (e.g. Ulwick et al., 1988). However, in recent years two experimental facts provided strong evidence against some parts of this theory: first, in situ measurements of neutral air turbulence in PMSE showed that neutral air turbulence regularly existed only in the upper altitude range with PMSE (Lübken et al., 2002). Secondly, in situ measurements of plasma parameters of the PMSE environment like number densities of electrons, positive ions and charged particles showed that in the majority of cases the charge residing 
on the particles is significantly less than the free electron number density (Havnes et al., 2001; Blix et al., 2003) contrary to the predictions of the Cho et al. (1992)-theory.

Recently, Rapp and Lübken (2003) proposed a possible solution for these two dilemmas: they reconsidered the question of electron diffusion in the vicinity of charged aerosol particles and found that in principle each perturbation of the negatively (positively) charged aerosol number density should also be reflected in anticorrelated (correlated) perturbations of the electron number density, independently of the actual ratio between charged particle number density and electron number density. Rapp and Lübken (2003) further showed that the lifetime of such electron perturbations is proportional to the square of the particle radius. Hence, fluctuations in the aerosol and electron density profiles which have initially been created by neutral air turbulence have the chance to prevail for periods of up to hours after the turbulent event has ceased. Such remnants of former turbulent activity are commonly termed "fossil"-turbulent structures (e.g. Cho et al., 1996).

In this paper we test these ideas by investigating the small scale structure of electron and particle charge number density observed in situ in the presence of PMSE. After a short description of the experiments and the general structure of the charged species observed in the PMSE altitude range we investigate in detail the degree of correlation between the small scale structures of electron and charged particle number densities and its relation to the actually observed radar scatter strength. Then we consider the shape of observed power spectra and we discuss these observations in the light of the new theory by Rapp and Lübken (2003). Finally, we close with a summary and recommendations for further work on this subject.

\section{Experimental technique}

The observations described in the current paper were obtained during sounding rocket flight ECT02 which was launched on 28 July 1994 at 22:39 UT. This rocket launch took place in the scope of the ECHO-campaign during which radar and lidar echoes from the polar summer mesopause region were studied with the ALOMAR-SOUSY VHF-radar (Bremer et al., 1996), with the ALOMAR and University of Bonn lidar (Lübken et al., 1996), and with sounding rockets.

The principal setup of the rocket payload is sketched in Fig. 1. Main instruments onboard the payload were a particle detector, named DUSTY, mounted on the top of the payload (provided by the University of Tromsø) and the combined sensor for neutrals and electrons, CONE, mounted in the rear of the payload (provided by the University of Bonn, Germany, and the Norwegian Defense Research Establishment). Note that all measurements presented in this study were obtained on the ascent part of the rocket trajectory since

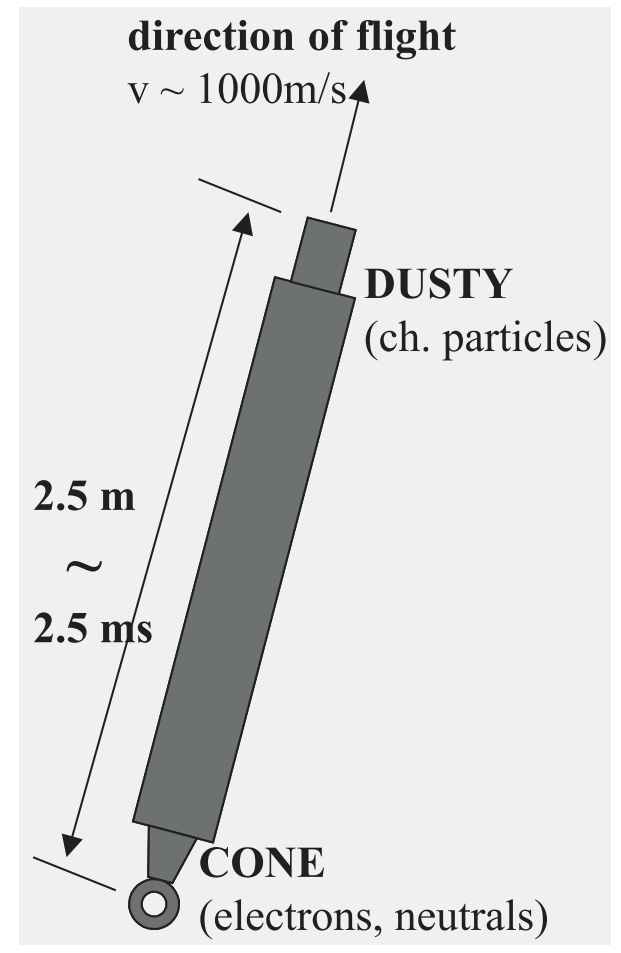

Fig. 1. Schematic of the rocket payload launched during flight ECT02. For a more detailed description of the instruments see the text.

the DUSTY particle detector needs to be in the ram position in order to detect particles.

The DUSTY instrument basically consists of a sensitive electrometer at the bottom of a bucket-like structure which is designed to measure both negative and positive currents due to charge resting on impacting aerosol particles. The primary measured quantity of the DUSTY experiment is hence a current which is proportional to $N_{A} Z_{A}$, i.e. the number density of heavy charged particles times the average charge of these particles. Using probe theory (e.g. Sagalyn et al., 1963; Smiddy and Stuart, 1969) the measured currents are converted to charge number densities of aerosol particles. For more details on the DUSTY instrument see the papers by Havnes et al. (1996a) and Havnes et al. (1996b).

The CONE-sensor is a combination of an ionization gauge for the measurement of neutral densities and a fixed biased Langmuir probe (bias potential $+6 \mathrm{~V}$ ) for the measurement of electron number densities. More details on the CONE instrument can be found in Giebeler et al. (1993) and Rapp et al. (2001). In this paper we focus on the electron measurements from the fixed biased Langmuir probe. As in the case of the DUSTY instrument, measured currents are converted to electron number densities applying standard probe theory (e.g. Sagalyn et al., 1963; Smiddy and Stuart, 1969). 


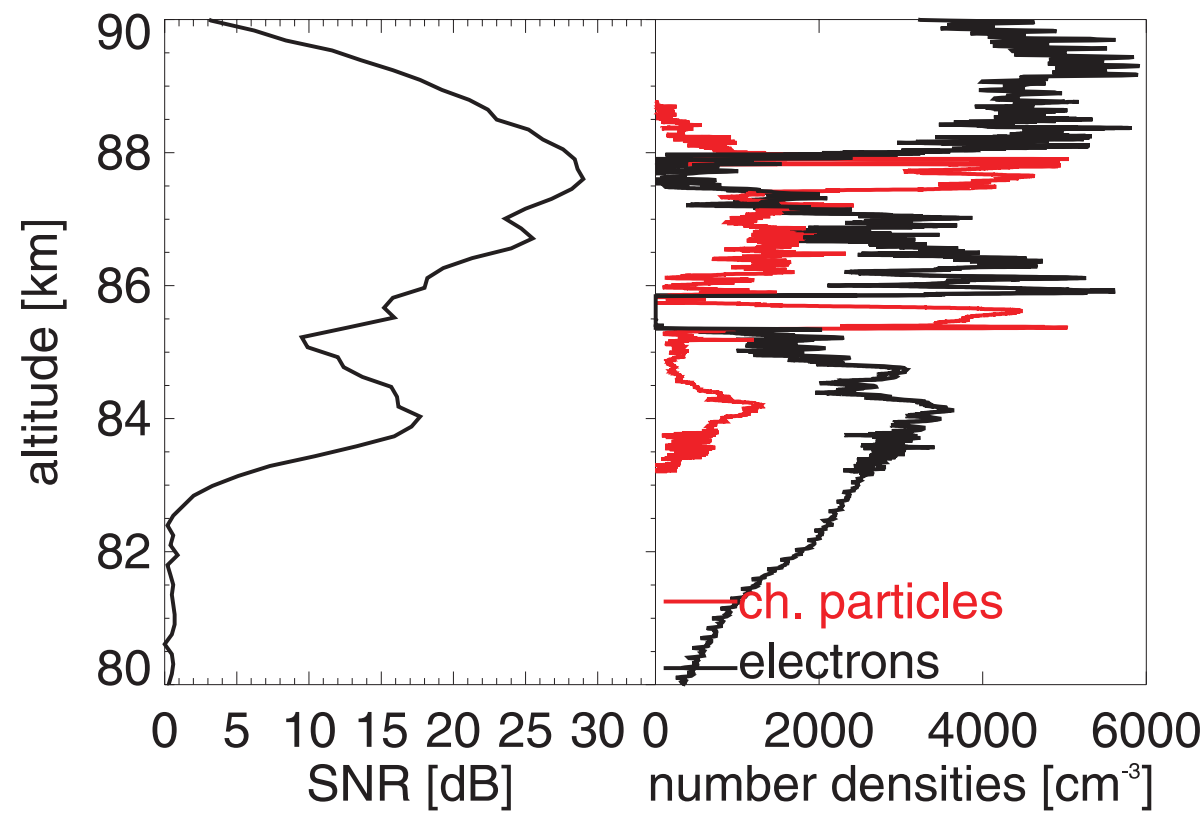

Fig. 2. Left panel: Profile of the radar signal to noise ratio (SNR) measured with the ALOMAR-SOUSY VHF radar during sounding rocket flight ECT02. Right panel: profiles of electron number densities (black line) and aerosol charge number densities (red line) measured during sounding rocket flight ECT02. These data are reproduced from Havnes et al. (1996b) and Lübken et al. (1998), copyright by the American Geophysical Union.

\section{Observations}

\subsection{General structure of observed plasma profiles}

In this section we start with a short summary of the main observed features during this rocket flight presented earlier (Havnes et al., 1996b,a; Lübken et al., 1998). In Fig. 2 we present measured profiles of electron number densities and charge number densities of heavy particles as well as the radar signal to noise ratio obtained by the ALOMARSOUSY VHF radar at the time of the rocket observations. As is evident from this figure, the DUSTY instrument observed negatively charged particles in the entire altitude range between 83 and $89 \mathrm{~km}$. The most remarkable features are two distinct layers of negatively charged particles centered at $\sim 88 \mathrm{~km}$ and $\sim 85.5 \mathrm{~km}$, respectively. At exactly the same altitudes, the electron number density profile measured by the CONE sensor showed two deep depletions, which is strong evidence that the aerosol particles become charged due to electron capture from the ambient D-region plasma (e.g. Rapp and Lübken, 2001).

\subsection{Small scale plasma variations}

Now we continue with a closer inspection of the small scale structures of the measured plasma density profiles. As pointed out in the introduction, the new PMSE theory by Rapp and Lübken (2003) predicts an anticorrelation of elec- trons and negatively charged particles (see also Lie-Svendsen et al., 2003).

In Fig. 3 we show two short time series of electron and charged particle fluctuations sampled at a frequency of $1250 \mathrm{~Hz}$ from altitudes of $\sim 88 \mathrm{~km}$ and $\sim 86.7 \mathrm{~km}$, respectively. The fluctuations have been obtained from the measurements shown in Fig. 2 by subtracting a background profile that was determined by a 3rd-order polynomial fit. Next, we have determined the cross-correlation of the observed fluctuations (shown in the lower two panels of Fig. 3). The cross-correlation determines the correlation coefficient of two time series for a given lag time between the two data sets. The cross-correlation analysis shows a maximum (negative) correlation at a lag time of $2.5 \mathrm{~ms}$. $2.5 \mathrm{~ms}$ is exactly the time period between the measurement of the DUSTY insrument and the CONE instrument at exactly the same place since the two instruments are separated by $2.5 \mathrm{~m}$ (=the payload length, see Fig. 1) and the rocket is moving at a speed of $\sim 1000 \mathrm{~m} / \mathrm{s}$ at these altitudes (see also Blix and Thrane, 1993). This proves that the fluctuations have not been artificially created by for example aerodynamic effects due to the supersonic motion of the rocket (e.g. Gumbel, 2001) or by payload charging effects (e.g. Holzworth et al., 2001) since such disturbances would need to occur at zero lag time.

Note also that the two instruments do not necessarily observe small scale irregularities in the entire altitude range with charged particles present. In Fig. 4a we show small scale variations of electrons and charged particles at altitudes 

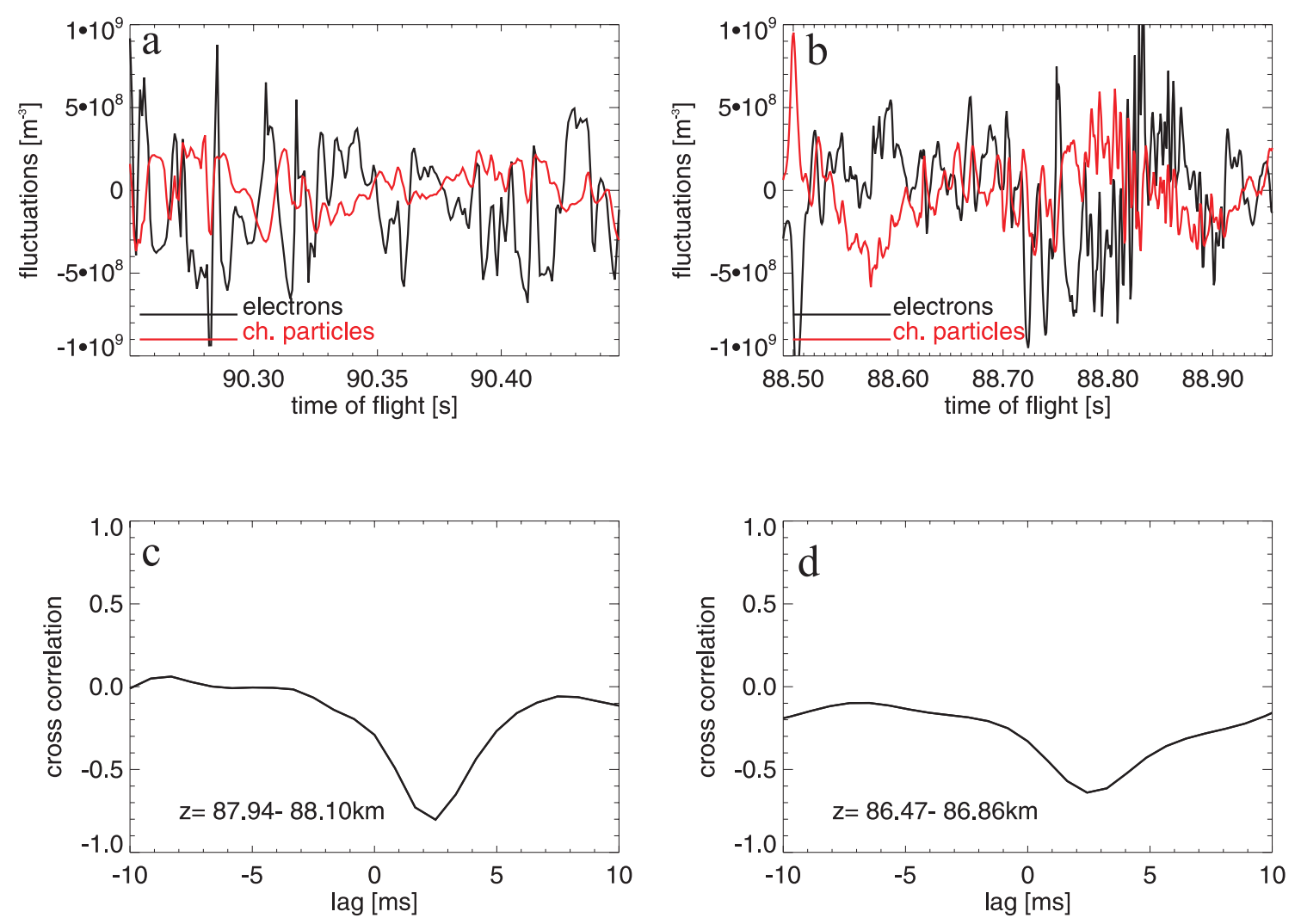

Fig. 3. Panels (a) and (b): Perturbations of electron (black line) and particle charge number densities (red line) during two selected time intervals. Panels (c) and (d): cross-correlation coefficients as a function of the time lag between electron and charged particle data for the data sets shown in Panel a and b, respectively.

between $84.6 \mathrm{~km}$ and $84.77 \mathrm{~km}$. At these altitudes, the background particle charge number density is $\sim 1000 / \mathrm{cm}^{3}$ but evidently almost no small scale variations are observed. Consequently, the cross-correlation analysis does not show any significant correlation of the considered data sets (see Fig. 4c).

Finally, we note one interesting feature of the discussed data set: at the lowermost altitudes with charged particles present, the small scale variations of charged particles and electrons indeed depart from the otherwise prevailing anticorrelation (see Figs. 4b, d). Here, in fact a strong positive correlation - again at a lag time corresponding to the rocket length - is observed. A possible explanation of such a feature was recently presented in the theoretical study by LieSvendsen et al. (2003) who proposed that a positive correlation could either occur in the presence of evaporating particles or in the presence of large and hence quickly sedimenting particles. A detailed analysis of this data set is currently conducted and will be presented in the near future.

\section{Comparison of radar and in situ measurements}

In this section we now consider the relation between the small scale plasma structures presented above and the ob- served radar signal. We follow Røyrvik and Smith (1984) who derived a relation between radar reflectivity and the power spectral densities of measured electron number density fluctuations at the radar Bragg scale. Essentially, the radar reflectivity $\eta$ is proportional to the power spectral density (PSD) of the absolute electron number density fluctuations at the radar half wavelength, i.e.,

$\eta \propto \tilde{\eta}_{e}=P S D\left(\Delta N_{e}, \lambda / 2\right)$,

where $\Delta N_{e}$ is a small scale random fluctuation on top of a smooth background profile. Since theory (Rapp and Lübken, 2003) predicts that $\Delta N_{e} \propto \Delta Z_{A} N_{A}$ where $\Delta Z_{A} N_{A}$ is a perturbation of the aerosol charge number density, $\eta$ should also be proportional to

$\tilde{\eta}_{A}=P S D\left(\Delta Z_{A} N_{A}, \lambda / 2\right)$.

In Fig. 5 we present a comparison of the radar signal to noise ratio measured during the sounding rocket flight with two profiles of $\tilde{\eta}_{e}$ and $\tilde{\eta}_{A}$, respectively. Power spectral densities of electron and charged aerosol number density fluctuations have been derived from data segments of $300 \mathrm{~m}$ in altitude obtained at a sampling frequency of $1250 \mathrm{~Hz}$ and a rocket velocity of $v_{R} \sim 1000 \mathrm{~m} / \mathrm{s}$. It turns out that the profiles of 

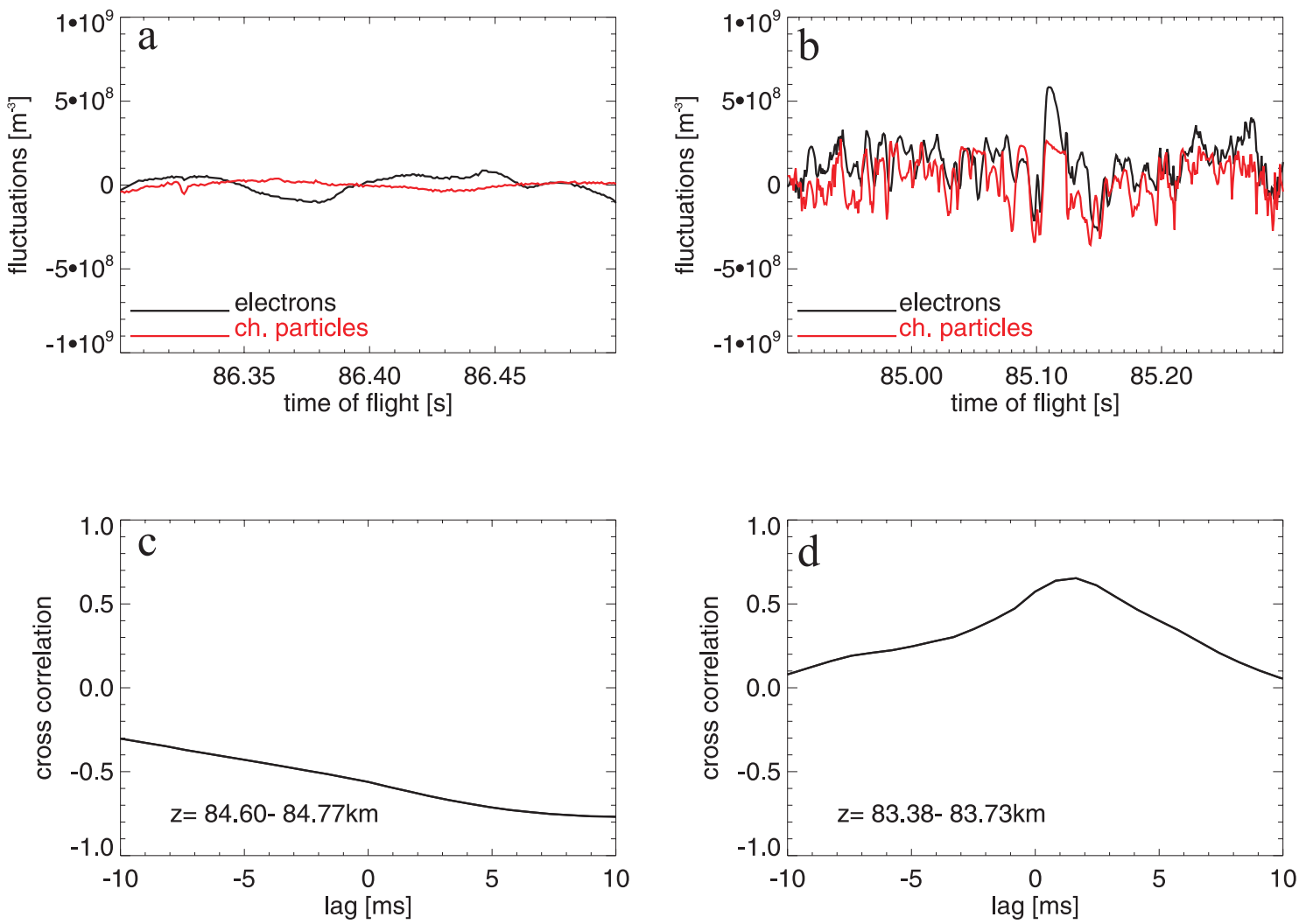

Fig. 4. Same as for Fig. 3 but for different time intervals.

$\tilde{\eta}_{e}$ and $\tilde{\eta}_{A}$ both resemble the actually measured radar signal very well. In general, both aerosol and electron fluctuations show the same behaviour, only at altitudes between 85 and $86 \mathrm{~km}$ we find that the derived signal due to electrons is zero whereas the signal due to fluctuations of charged particles qualitatively agrees with the actually observed radar signal. The reason for this discrepancy can be understood if we look at the altitude profiles of charged particles and electrons shown in Fig. 2. Between 85 and $86 \mathrm{~km}$, the aerosol charge number density is maximum thereby leading to an almost complete biteout in the electron number density since the aerosol particles become charged due to the capture of electrons from the background atmosphere (e.g. Rapp and Lübken, 2001).

In this context, the natural question arises why we observe a radar signal from altitudes where the in situ measured electron number density is zero. Here, we have to consider that the radar signal is received from an atmospheric volume of $\sim 10 \mathrm{~km}$ in diameter (=the beam width) and $300 \mathrm{~m}$ in thickness (=one radar range gate) whereas the rocket only yields "point"-measurements (i.e., typical probe dimensions are 5$10 \mathrm{~cm}$ ). Hence, in the case that the electron number density is horizontally structured it can happen that the rocket measurement observes a local spot with zero electron number density whereas the radar sees an average signal from the entire probed volume where other patches with non-zero electron number densities exist. Such horizontal structuring in the electron number density could for example be created by tilted ice particle layers that become charged by electron capture and hence create tilted electron depletion layers, or it could be due to Kelvin-Helmholtz structures also acting on the charged ice particle layers (Gumbel, 2003). In spite of this deficiency, rocket borne measurements of the electron number density yield so far the best possible characterization of the radar refractive index at these altitudes and are hence a useful tool to test existing theories like it is done in the current paper. In our case, this statement is certainly supported by the overall good agreement between radar SNR and the derived profiles of $\tilde{\eta}_{e}$ and $\tilde{\eta}_{A}$. Nevertheless, we note that a characterization of the horizontal structure of the electron number density would be highly desirable for future PMSE research.

In summary, the observed radar signal is directly reflected in the small scale structure of both aerosol particles and electrons. This result is consistent with the idea that the electron fluctuations are indeed a direct consequence of the perturbations in the charged aerosol particle distribution (Cho et al., 1992; Rapp and Lübken, 2003). 


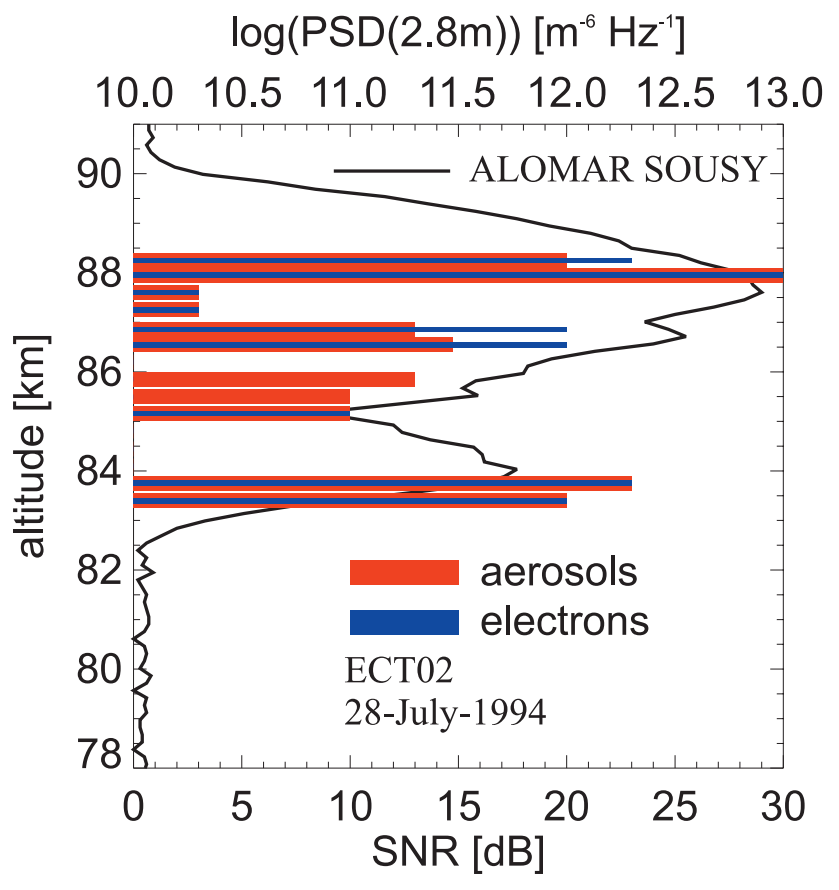

Fig. 5. Comparison of the radar signal to noise ratio, SNR, during the sounding rocket flight ECT02 (black line, lower abscissa) and $P S D\left(\Delta N_{e}, \lambda / 2\right)$ and $P S D\left(\Delta N_{A}, \lambda / 2\right)$ (red bars for charged aerosol particles, blue bars for electrons, upper abscissa).

\section{The spectral shape of electron and charged particle fluctuations in PMSE}

\subsection{Model estimate}

In this section we consider the spectral shape of the fluctuations of aerosol particles and electrons that we expect due to the theoretical ideas proposed by Rapp and Lübken (2003). The main idea is that neutral air turbulence initially creates the small scale structures of the plasma species that extend to scales much smaller than the smallest scales in the neutral gas because of their reduced diffusivities. The spectral shape of the power spectrum for such low diffusivity tracers was predicted by Batchelor (1959) and later confirmed by laboratory measurements (Gibson and Schwartz, 1963) and sophisticated direct numerical simulation methods (Bogucki et al., 1997). According to Batchelor's theory the three dimensional power spectrum takes the following form for spatial scales smaller than the Kolmogoroff-microscale $l_{K}=\left(v^{3} / \epsilon\right)^{\frac{1}{4}}$ :

$\Gamma(k)=\frac{2 \chi}{k} \sqrt{\frac{\nu}{\epsilon}} \cdot \exp \left(-2 l_{B}^{2} k^{2}\right)$

where $\chi$ is the rate at which fluctuations of the tracer are dissipated, $\mathrm{k}$ is the wavenumber, $l_{B}=\left(v D^{2} / \epsilon\right)^{\frac{1}{4}}$ is the so-called Batchelor-scale (where $\mathrm{D}$ is the diffusion coefficient of the tracer), $\epsilon$ is the turbulent energy dissipation rate, and $v$ is the

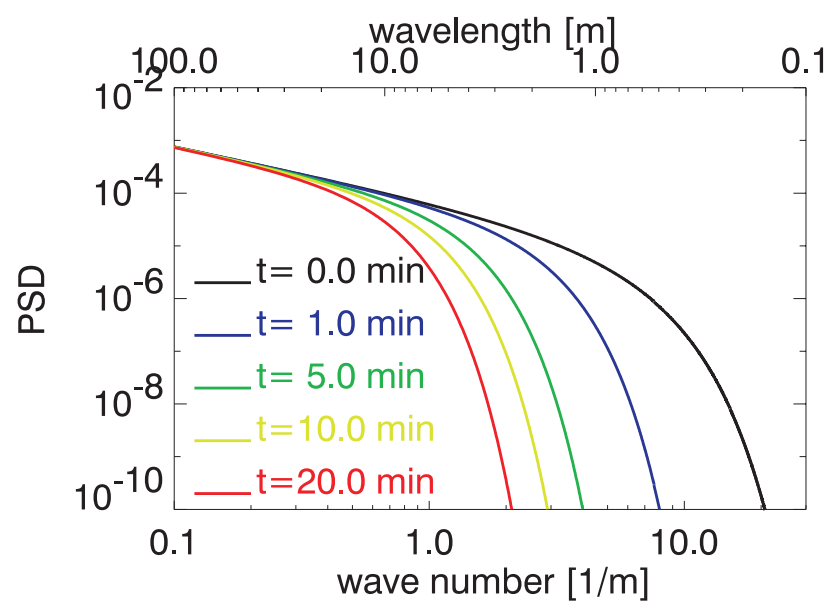

Fig. 6. Power spectra of tracer variance according to Eq. (3) and (7) for a turbulent energy dissipation rate $\epsilon=0.1 \mathrm{~m}^{2} / \mathrm{s}^{3}$, kinematic viscosity $\nu=1.5 \mathrm{~m}^{2} / \mathrm{s}$, an aerosol radius $r_{A}=10 \mathrm{~nm}$, and different times after active neutral air turbulence stopped (color coded).

kinematic viscosity of air. This expression yields the classical $k^{-1}$-dependence for scales between the Kolmogorowmicroscale and the Batchelor-scale characterizing the socalled viscous-convective subrange, and an exponential decay for larger wavenumbers. For the comparison with spectra derived from rocket measurements, however, the three dimensional power spectrum provided by Eq. (3) must be converted to a one dimensional spectrum, i.e. measured along a rocket trajectory. As explained in Bogucki et al. (1997) this yields

$$
\begin{array}{r}
\Gamma(k)_{1 d}=\frac{2 \chi}{k} \sqrt{\frac{\nu}{\epsilon}} \cdot \exp \left(-2 l_{B}^{2} k^{2}\right) \\
+\sqrt{8 \pi} \chi \sqrt{\frac{\nu}{\epsilon}} \cdot l_{B} \cdot\left(\operatorname{erf}\left(\sqrt{2} l_{B} k\right)-1\right)
\end{array}
$$

In Fig. 6 we show such a spectrum for fluctuations of charged aerosol particles with a radius of $10 \mathrm{~nm}$, a typical turbulent energy dissipation rate $\epsilon=0.1 \mathrm{~m}^{2} / \mathrm{s}^{3}$ and $\nu=1.5 \mathrm{~m}^{2} / \mathrm{s}$. From the aerosol radius, the diffusion coefficient of charged particles can be roughly estimated as $D_{A}=v /\left(6.5 \cdot r_{A}^{2}\right)$ where $\mathrm{r}_{A}$ is given in $\mathrm{nm}$ (e.g. Lübken et al., 1998). Now, we consider how this spectral shape is expected to change when the turbulent event stops and the fluctuations decay. In order to estimate the temporal behaviour of $\Gamma(k)$ we assume that the fluctuations change only because of diffusion. For simplicity we also assume that both electrons and charged aerosol particles decay with the diffusion coefficient of the aerosols $D_{A}$ (see Rapp and Lübken, 2003, for a justification of this simplification). Then, each Fourier-component $A(k, x)=\tilde{\Gamma}(k) \cdot \cos \left(k \cdot x+\phi_{k}\right)$ (where $x$ is the spatial coordinate, $\phi_{k}$ is a random phase and $\tilde{\Gamma}(k)$ is the power spectrum at the time just before the active turbulence ceased) of the 

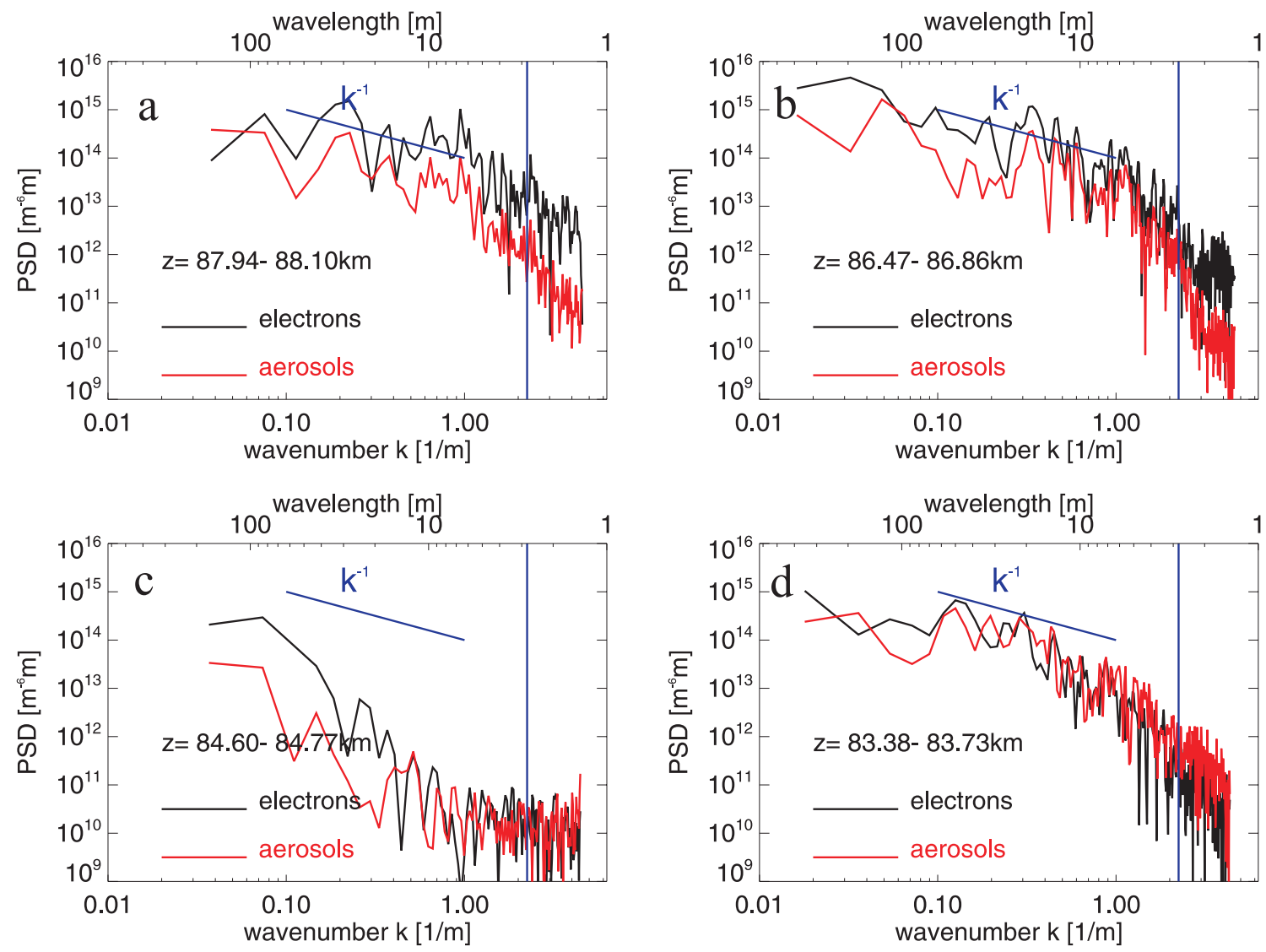

Fig. 7. Power spectra of electron number density (black lines) and particle charge number density fluctuations (red lines) shown in the Figs. 3 and 4 , respectively. Note that principally the data presented have been measured in the time rather than in the spatial domain. Wavenumbers have been determined from measured frequencies $f$ applying the relation $k=2 \pi f / v_{R}$ where $v_{R}$ is the rocket velocity $(\sim 800 \mathrm{~m} / \mathrm{s}$ in our cases). The inclined line represents a spectral slope of -1 and the vertical line marks the radar Bragg scale $(=2.8 \mathrm{~m})$.

spatial distribution of charged aerosols and electrons must obey a diffusion equation, i.e.

$\frac{\partial A}{\partial t}=D_{A} \frac{\partial^{2}}{\partial x^{2}} A$,

which is solved by

$$
A(k, x, t)=\underbrace{\tilde{\Gamma}(k) \cdot \cos \left(k \cdot x+\phi_{k}\right)}_{A(k, x)} \cdot e^{-D_{A} k^{2} t}
$$

Note that this solution implies that during the diffusional decay there is no transfer of power spectral density from one Fourier mode to the other. This is evident from Eq. (6) which shows that only the amplitude and not the wavenumber (or wavelength) of $A$ changes with time. Hence, the power spectrum is expected to vary like

$\Gamma(k, t)=\tilde{\Gamma}(k) \cdot e^{-D_{A} k^{2} t}$

In Fig. 6 we show an example for the decay of an initial Batchelor-spectrum after Eqs. (4) and (7) for decay times between 1 and twenty minutes. Evidently, the general spectral shape of the Batchelor spectrum is preserved, however, the part of the spectrum showing a $k^{-1}$ dependence shrinks in favor of the steeper, exponentially decaying spectrum. Note that the decay time of the spectral subrange with a slope of -1 is directly proportional to $D_{A}$ which is proportional to $1 / r_{A}^{2}$ (see above). Hence, in the presence of rather large aerosol particles, spectra of electrons and charged aerosol particles may exhibit a subrange with a slope of -1 even a considerable time after turbulence has ceased.

In summary, spectra of both electron number density and particle charge number density fluctuations should reveal a viscous convective subrange with its characteristic spectral slope of -1 . Importantly, this should happen both in the case of active as well as fossil turbulence provided that only diffusion leads to the destruction of fluctuations after the end of active turbulence. In the next subsection we will investigate if spectra determined from observed fluctuations indeed show this behaviour. 


\subsection{Observed spectra}

In Fig. 7 we show the power spectra of the electron and charged particle fluctuations shown in Figs. 3 and 4, respectively. Note that we only consider data segments away from the edges of the electron biteouts since the sharp edges occurring there can significantly influence the spectral shape see Alcala et al. (2001) for more details. These spectra show several striking features: first of all and most striking, the spectra of electrons and charged aerosol particles are very similar both regarding the shape and the absolute values of observed power spectral densities. In addition, in all the three cases where strong fluctuations were observed, i.e. shown in Figs. 7a, b, d, the spectra show indeed spectral ranges with a slope very similar to $\sim-1$, indicating the presence of a viscous convective subrange. The spectra shown in Fig. 7c show only white noise for wavenumbers larger than $\mathrm{k}=0.5 \mathrm{~m}^{-1}$ as expected from the fluctuations shown in Fig. 4a.

One cautionary note must be added here: due to the fact that the diffusional decay of the plasma irregularities does not alter the general spectral shape, it turns out that it is not possible to judge about the state of turbulence, i.e. if it is active or if only fossil remnants are observed, just from the observed spectra. This requires an independent measurement, e.g. the measurement of neutral air density fluctuations with the ionization gauge part of the CONE sensor. Unfortunately, these observations can only be conducted when the CONE sensor is in ram position during the downleg part of the rocket trajectory (whereas all the observations presented here were obtained on the upleg part of the rocket trajectory). As described in Lübken et al. (2002), the CONE sensor identified turbulent activity at altitudes between 87.5 and $90.5 \mathrm{~km}$ and between 92.5 and $93.5 \mathrm{~km}$ on the downleg part of the trajectory. If we would assume that the turbulence is homogeneous over the distance between upleg and downleg of the rocket trajectory (horizontal distance $\sim 50 \mathrm{~km}$ ), we could classify the spectrum shown in Fig. 7a as "active" whereas the spectra shown in the Figs. 7b and d would be "fossil". However, since neutral air turbulence measurements in exactly the same volume are not available we admit that these statements must be considered with care.

\section{Conclusions}

We summarize our findings as follows:

1. Fluctuations of electrons and charged particles show a prominent anticorrelation during most of the observed particle layer where small scale fluctuations are present. Only at the lowermost altitudes the correlation turns positive. See the recent investigation of Lie-Svendsen et al. (2003) for a detailed discussion of such cases.
2. Comparing the power spectral density of these fluctuations at the Bragg scale with the observed radar signal to noise profile shows general agreement.

3. The observed spectra of both electrons and charged particles show an inertial-viscous subrange as theoretically expected on the grounds of Batchelor (1959) and Rapp and Lübken (2003) for the cases of active and fossil turbulence acting on low diffusivity tracers, respectively.

All these results are consistent with the theoretical ideas by Rapp and Lübken (2003) suggesting that PMSE can be explained by a combination of active and fossil neutral air turbulence acting on the large and heavy charged aerosol particles which are subsequently mirrored in the electron number density distribution that becomes visible to a VHF radar when small scale fluctuations are present.

However, further experimental and theoretical work should be performed to corroborate (or falsify) this approach. E.g. on the theoretical side, more sophisticated calculations such as a direct numerical simulation of the physical processes (like e.g. the approach by Hill et al., 1999) also after the end of neutral air turbulence would be highly desirable. Experimentally, an in situ experiment where fluctuations of electrons, charged particles, positive ions, and the neutral gas are measured in the same volume could yield more precise information allowing to classify observed plasma spectra as evidence of "active" or "fossil" turbulence.

Acknowledgements. We are indebted to O. Havnes, University of Troms $\emptyset$, for kindly providing the charged particle data obtained with the DUSTY instrument. The rocket flight ECT02 was supported by the Bundesministerium für Bildung, Wissenschaft, Forschung und Technologie under DARA grant 01OE88027.

\section{References}

Alcala, C. M., Kelley, M. C., and Ulwick, J. C.: Nonturbulent layers in polar summer mesosphere 1. detection of sharp gradients using wavelet analysis, Radio Sci., 36, 875-890, 2001.

Batchelor, G. K.: Small-scale variation of convected quantities like temperature in a turbulent fluid, J. Fluid Mech., 5, 113-133, 1959.

Blix, T. A. and Thrane, E.: Noctilucent clouds and regions with polar mesopheric summer echoes studied by means of rocket-borne electron and ion DC-probes, Geophys. Res. Lett., 20, 23032306, 1993.

Blix, T. A., Rapp, M., and Lübken, F.-J.: Relations between small scale electron number density fluctuations, radar backscatter and charged aerosol particles, J. Geophys. Res., 108, D8, 8450, doi:10.1029/2002JD002430, 2003.

Bogucki, D., Domaradzki, J. A., and Yeung, P. K.: Direct numerical simulations of passive scalars with $\operatorname{Pr}>1$ advected by turbulent flow, J. Fluid Mech., 343, 111-130, 1997.

Bremer, J., Hoffmann, P., Singer, W., Meek, C., and Rüster, R.: PMSE observations with the ALOMAR-SOUSY and the EISCAT-VHF radar during the ECHO-94 campaign, Geophys. Res. Lett., 23, 1075-1078, 1996. 
Cho, J. Y., Alcala, C. M., Kelley, M. C., and Swartz, W. E.: Further effects of charged aerosols on summer mesospheric radar scatter, J. Atmos. Terr. Phys., 58, 661-672, 1996.

Cho, J. Y. N., Hall, T. M., and Kelley, M. C.: On the role of charged aerosols in polar mesosphere summer echoes, J. Geophys. Res., 97, 875-886, 1992.

Ecklund, W. L. and Balsley, B. B.: Long-term observations of the arctic mesosphere with the MST radar at Poker Flat, Alaska, J. Geophys. Res., 86, 7775-7780, 1981.

Gibson, C. H. and Schwartz, W. H.: The universal equilibrium spectra of turbulent velocity and scalar fields, J. Fluid Mech., 16, 365-384, 1963.

Giebeler, J., Lübken, F.-J., and Nägele, M.: CONE - a new sensor for in-situ observations of neutral and plasma density fluctuations, Proceedings of the 11th ESA Symposium on European Rocket and Balloon Programmes and Related Research, Montreux, Switzerland (ESA SP), ESA-SP-355, 311-318, 1993.

Gumbel, J.: Aerodynamic influences on atmospheric in situ experiments on sounding rockets, J. Geophys. Res., 106, 10553 $10563,2001$.

Gumbel, J.: Interactive comment on "Small scale density variations of electrons and charged particles in the vicinity of polar mesosphere summer echoes" by M. Rapp et al., Atmos. Chem. Phys. Discuss., 3, 1372-1373, 2003.

Havnes, O., Næsheim, L. I., Hartquist, T., Morfill, G. E., Melands $\varnothing$, F., Schleicher, B., Trøim, J., Blix, T., and Thrane, E.: Meter-scale variations of the charge carried by mesospheric dust, Planet. Space Sci., 44, 1191-1194, 1996a.

Havnes, O., Trøim, J., Blix, T., Mortensen, W., Næsheim, L. I., Thrane, E., and Tønnesen, T.: First detection of charged dust particles in the Earth's mesosphere, J. Geophys. Res., 101, 10839 $10847,1996 \mathrm{~b}$.

Havnes, O., Brattli, A., Aslaksen, T., Singer, W., Latteck, R., Blix, T., Thrane, E., and Trøm, J.: First common volume observations of layered plasma structures and polar mesospheric summer echoes by rocket and radar, Geophys. Res. Lett., 28, 1419-1422, 2001.

Hill, R. J., Gibson-Wilde, D. E., Werne, J. A., and Fritts, D. C.: Turbulence-induced fluctuations in ionization and application to PMSE, Earth Plan. Space, 51, 499-513, 1999.

Holzworth, R., Pfaff, R., Goldberg, R., Bounds, S., Schmidlin, F., Voss, H., Tuzzolino, A., Croskey, C., Mitchell, J., von Cossart, G., Singer, W., Hoppe, U.-P., Murtagh, D., Witt, G., Gumbel, J., and Friedrich, M.: Large electric potential perturbations in PMSE during DROPPS, Geophys. Res. Lett., 28, 1435-1438, 2001.
Kelley, M. C., Farley, D. T., and Röttger, J.: The effect of cluster ions on anomalous VHF backscatter from the summer polar mesosphere, Geophys. Res. Lett., 14, 1031-1034, 1987.

Lie-Svendsen, O., Blix, T. A., Hoppe, U., and Thrane, E.: Modelling the plasma response to small-scale particle perturbations in the mesopause region, J. Geophys. Res., 108, D8, 8442,doi:10.1029/2002JD002753, 2003.

Lübken, F.-J., Fricke, K.-H., and Langer, M.: Noctilucent clouds and the thermal structure near the Arctic mesopause, J. Geophys. Res., 101, 9489-9508, 1996.

Lübken, F.-J., Rapp, M., Blix, T., and Thrane, E.: Microphysical and turbulent measurements of the Schmidt number in the vicinity of polar mesosphere summer echoes, Geophys. Res. Lett., 25, 893-896, 1998.

Lübken, F.-J., Rapp, M., and Hoffmann, P.: Neutral air turbulence and temperatures in the vicinity of polar mesosphere summer echoes, J. Geophys. Res., 107(D15), doi:10.1029/2001JD000915, 2002.

Rapp, M. and Lübken, F.-J.: Modelling of particle charging in the polar summer mesosphere: Part 1 - general results, J. Atmos. Sol. Terr. Phys., 63, 759-770, 2001.

Rapp, M. and Lübken, F.-J.: On the nature of PMSE: electron diffusion in the vicinity of charged particles revisited, J. Geophys Res., 108, D8, 8437, doi: 10.1029/2002JD002857, 2003.

Rapp, M., Gumbel, J., and Lübken, F.-J.: Absolute density measurements in the middle atmosphere, Ann. Geophys., 19, 571-580, 2001.

Røyrvik, O. and Smith, L. G.: Comparison of mesospheric VHF radar echoes and rocket probe electron concentration measurements, J. Geophys. Res., 89, 9014-9022, 1984.

Sagalyn, R. C., Smiddy, M., and Wisnia, J.: Measurements and interpretation of ion density distributions in the daytime F-region, J. Geophys. Res., 68, 199-211, 1963.

Smiddy, M. and Stuart, R. D.: Phys. Sci. Res. Papers 364, AFCRL69-0013, Office of Aerospace Research, Hanscom Field, Bedfort, Mass., 1969.

Ulwick, J. C., Baker, K. D., Kelley, M. C., Balsley, B. B., and Ecklund, W. L.: Comparison of simultaneous MST radar and electron density probe measurements during STATE, J. Geophys. Res., 93, 6989-7000, 1988.

von Zahn, U. and Bremer, J.: Simultaneous and common-volume observations of noctilucent clouds and polar mesosphere summer echoes, Geophys. Res. Lett., 26, 1521-1524, 1999. 